\title{
Laparoscopic resection of giant retroperitoneal schwanomma located in left supra renal area: a rare case report
}

\begin{abstract}
Schwannomas are rare tumors originating from the Schwann sheath of peripheral or cranial nerves. They are usually benign tumors and in $95 \%$ of cases they originate from peripheral nerves. Occurrence in retroperitoneum is very rare. We report a case of giant retroperitoneal schwannoma by laparoscopy removal in 59year old male, moderately obese who presented with vague abdominal discomfort of 14 years duration, evaluated outside as Giant Retroperitoneal tumor of size $17 * 15 * 12 \mathrm{~cm}$ in the left supra renal area. The pathologic diagnosis was schwannoma. Successful management of such a Giant Retroperitoneal schwannoma completely by laparoscopy is the first documented case in the literature.
\end{abstract}

Keywords: retroperitoneal tumor, schwannoma, laparoscopy
Volume 9 Issue 2 - 2019

\author{
Mallikarjuna N Reddy, ' Ajay Kumar G,' \\ Kalyan Koti, ${ }^{2}$ Prasad Babu BSR, ${ }^{3}$ Lavakumar \\ $A,{ }^{4}$ Sindhuri $A^{5}$ \\ 'Department of Urology, Sentini Hospitals, India \\ ${ }^{2}$ Department of Pathology, Sentini Hospitals, India \\ ${ }^{3}$ Department of Radio-Diagnosis, Sentini Hospitals, India \\ ${ }^{4}$ Department of Anaesthesiology, Sentini Hospitals, India \\ ${ }^{5}$ Department of Pulmonology, Sentini Hospitals, India
}

Correspondence: Ajay Kumar G, Department of Urology, Sentini Hospitals, India, Email drajay707@gmail.com

Received: February 26, 2019 | Published: March 21, 2019

\section{Introduction}

Primary tumors of the retroperitoneal region are quiet rare, and schwannomas comprise only $1-10 \%$ of them. Schwannomas originate from Schwann cells of the peripheral nerve fibers and are usually located in the head, neck, and flex or surfaces of the extremities. Most of the schwannomas are asymptomatic and found accidently. Microscopically, positive expression of S-100 and negative expression of CD 34 is valuable for diagnosis. Here in, we report a rare case of Retroperitoneal Schwannom successfully resected completely by laparoscopy procedure.

\section{Case presentation}

A 59-year-old man weighting $102 \mathrm{~kg}$, presented with vague abdominal discomfort in the left side of abdomen of 14years duration. He described it as, on and off in nature. Other constitutional symptoms were, less than $10 \%$ weight loss noted. He is Type 2DM. No significant past surgical history. His abdominal physical exam was normal. On laboratory tests, evaluation for pheochromocytoma was done and all parameters were b within normal limits. Computed Tomorgraphic Scan with non-ionic contrast showed a solid well-differentiated heterogeneous mass, $17 \mathrm{~cm} \times 15 \mathrm{~cm} \times 12 \mathrm{~cm}$, located between the left kidney, spleen and aorta (Figure 1).

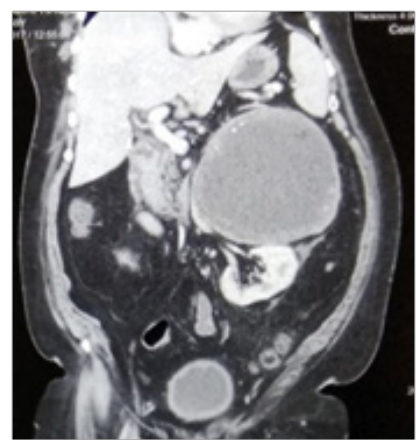

Figure I Computed Tomorgraphic Scan with non-ionic contrast showed solid well-differentiated heterogeneous mass, $17 \mathrm{~cm} \times 15 \mathrm{~cm} \times 12 \mathrm{~cm}$, located between the left kidney, spleen and aorta.
Under General Anaesthesia, the patient was placed in right semi lateral position. A primary $10 \mathrm{~mm}$ port placed supraumbilically for a telescope. A $10 \mathrm{~mm}$ port placed in the left midclavicular line. Two $5 \mathrm{~mm}$ ports placed in the midclavicular line and in the sub xiphisternal region for working elements. A monitor, $\mathrm{Co}_{2}$ pneumoperitoneum $(12 \mathrm{mmHg}$ ), a laparoscope (HD KARL STORTZ,GERMANY), an ultrasonic dissector, Thunder beat (OLYMOPUS,GERMANY) and routine laparoscopic instruments were used. The giant retroperitoneal mass seen pushing left kidney downwards and bowels anteriorly. Retroperitoneal approach was palnned. The descending colon is dissected and brought medially. The left suprarenal area is started dissecting out very carefully. All around the mass dissection done and freed from surrounding structures .Artery and vein to the mass identified separately, clipped and ligated (Figure 2). Retroperitoneal mass specimen was brought out into from the left side of abdomen, infraumbilically. Haemostasis secured. Drain placed and fixed. Port closure done. Postoperative recovery was uneventful and on fifth post op day, he was discharged from the hospital with complete recovery. The pathological analysis demonstrated Spindle cell neoplasm composed of alternatively hypo cellular areas with marked nuclear palisading, well formed verocay body and hyper cellular areas with edema (Figure 3A-C). Immuno Histo Chemical staining clarified that the neoplastic cells were strongly positive for s100 protein (Figure 3D), while CD117(C-Kit), SMA (Smooth Muscle Actin), Desmin, PDGFR (Platelet Derived Growth Factor Receptor) were negative.

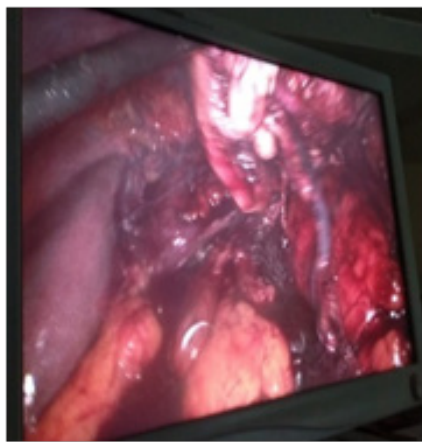

Figure 2 Artery and vein to the mass identified separately, clipped and ligated. 


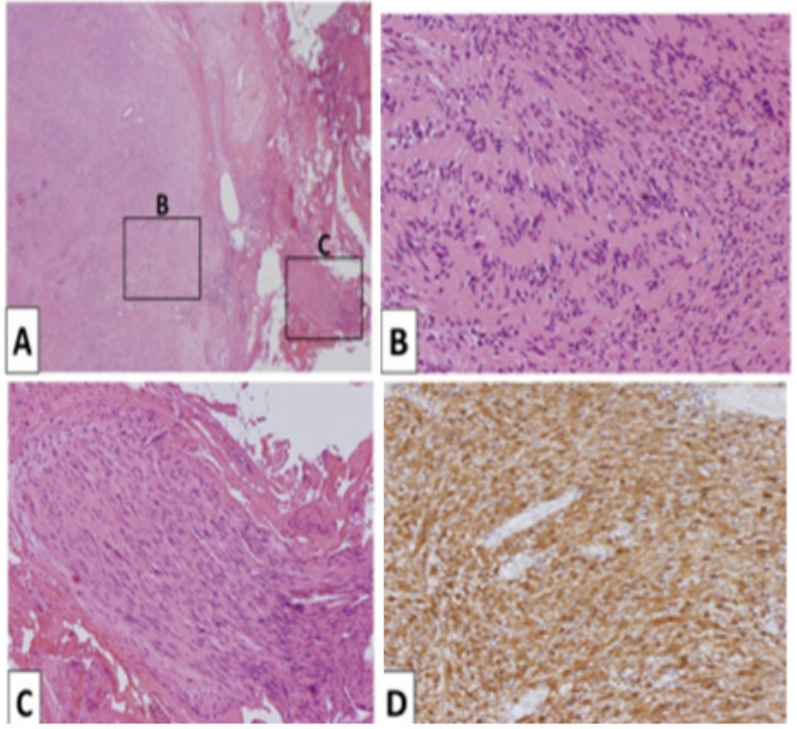

Figure 3 Microscopic findings. (A) A fascicular arrangement of strand spindle cells. (B) Palisading pattern. (C) A normal peripheral nerve entering into the tumor. (D) Immunohistochemical stains were positive for S- 100.

\section{Discussion}

Schwannomas are nerve sheath tumors that are mostly benign in nature. These neoplasms are usually seen in adult population between the ages of 20 and 50. Symptomatology of benign schwannomas is highly non specific and depends on the location and size of the lesion. Retroperitoneal region is a rare location for schwannomas except in patients having Von Recklinghausen's disease. It is also note worthy to mention that malignant degeneration particularly takes place in association with Von Recklinghausen's disease. In general, since the retroperitoneal space is rather large and flexible, the diagnosis of retroperitoneal schwannomas is often delayed, and the lesion reaches a significant size at the time of diagnosis. The most common symptoms are abdominal pain and distention.

The occurrence of a retroperitoneal schwannoma is uncommon comprising only $1-3 \%$ of all schwannoma and almost $1 \%$ of retroperitoneal neoplasms. ${ }^{1}$ Schwannomas are usually benign, slowgrowing, encapsulated tumors, and are rarely malignant. Malignant schwannomas are frequently associated with von Recklinghausen syndrome or other types of neurofibromatosis. In our case, the mass was so large that it occupied much space of the abdominal cavity and closely adhered to the adjacent vessels and organs which made the origination of the mass very difficult to diagnose by CT scan or B-ultrasound. Even during the operation, we thought the mass was from right kidney or adrenal gland when separated from the liver at the beginning. Pathologically, it is a well-encapsulated lesion demonstrating specific Antoni $\mathrm{A} / \mathrm{B}$ areas. Positive expression of S-100 and negative expression of CD 34 is valuable for diagnosis. ${ }^{2}$

The treatment of choice for retroperitoneal schwannoma is complete surgical resection ${ }^{3}$ particularly in those without expected response to chemo radiotherapy. Some authors believe that complete resection is ideal, an extensive operation sacrificing of vital structures may be warranted in order to achieve negative margins. ${ }^{4}$ In patients who underwent partial resection, some investigators have even reported recurrencerates of up to $10-20 \%$ even in benign circumstances, thus emphasizing the importance of a complete resection. ${ }^{5}$

\section{Conclusion}

Successful management of such a giant Retroperitoneal schwannoma completely by laparoscopy is the first documented case in the literature. Great expertise is required in the laparoscopic removal. High index of suspicion for the diagnosis is required.

\section{Acknowledgments}

None.

\section{Conflicts of interest}

The authors declared no potential conflicts of interest.

\section{References}

1. Dede M, Yagci G, Yenen MC, et al. Retroperitoneal benign schwannoma: report on three cases and analysis of clinic radiologic findings. Tohoku $J$ Exp Med. 2003;200:93-97.

2. Hughes MJ, Thomas JM, Fisher C, et al. Imaging features of retroperitoneal and pelvic schwannomas. Clin Radiol. 2005;60(8):886893.

3. $\mathrm{Gu} \mathrm{L}, \mathrm{Liu} \mathrm{W}, \mathrm{Xu} \mathrm{Q}$, et al. Retroperitoneal schwannoma mimicking hepatic tumor. Chin Med J. 2008;121(17):1751-1752.

4. Giglio M, Giasotto V, Medica M, et al. Retroperitoneal ancient schwannoma: case report and analysis of clinicoradiological findings. Annales d'urologie. 2002;36(2):104-106.

5. Tortorelli AP, Rosa F, Papa V, et al. Retroperitoneal schwannomas: diagnostic and therapeutic implications. Tumori. 2007;93(3):312-315. 